\title{
Intestinal Epithelium-Derived Galectin-9 Is Involved in the Immunomodulating Effects of Nondigestible Oligosaccharides
}

\author{
Sander de Kivit ${ }^{a} \quad$ Aletta D. Kraneveld $^{a}$ Leon M.J. Knippels ${ }^{a, b}$ \\ Yvette van Kooyk ${ }^{c}$ Johan Garssen ${ }^{a, b}$ Linette E.M. Willemsen ${ }^{a}$ \\ aDivision of Pharmacology, Utrecht Institute of Pharmaceutical Sciences, Faculty of Science, Utrecht University, \\ Utrecht, ${ }^{b}$ Danone Research, Center for Specialized Nutrition, Wageningen, and ${ }^{\mathrm{c} M o l e c u l a r}$ Cell Biology and \\ Immunology, Free University Medical Center, Amsterdam, The Netherlands
}

\section{Key Words}

Galectin-9 · Intestinal epithelial cells · Nondigestible oligosaccharides $\cdot$ Regulatory $T$ cells $\cdot T_{h} 1$ cells $\cdot$ TLR9

\begin{abstract}
Dietary intervention using nondigestible oligosaccharides, short-chain galacto-oligosaccharides (scGOS)/long-chain fructo-oligosaccharides (IcFOS), in combination with Bifidobacterium breve $\mathrm{M}-16 \mathrm{~V}$ prevents allergic disease involving galectin-9. In addition, apical TLR9 signaling contributes to intestinal homeostasis. We studied the contribution of galectin-9 secreted by intestinal epithelial cells (IEC; HT-29 and T84) in $T_{h} 1$ and regulatory $T$-cell $\left(T_{\text {reg }}\right)$ polarization in vitro. IEC were grown in transwell filters, cocultured with CD3/ CD28-activated human peripheral blood mononuclear cells (PBMC) and apically exposed to genomic DNA derived from B. breve M-16V or synthetic TLR9 ligand in the absence or presence of scGOS/lcFOS. Cytokine production and T-cell phenotype were determined and galectin expression by IEC was assessed. Galectin-9 was neutralized using lactose or a TIM-3-Fc fusion protein. IEC exposed to DNA from B. breve $M-16 V$ or TLR9 ligand in the presence of scGOS/lcFOS enhanced IFN- $\gamma$ secretion by PBMC and increased the percentage of $T_{h} 1$ and $T_{\text {reg }}$ cells. Expression and secretion of galec-
\end{abstract}

tin- 9 by IEC was increased and neutralization of galectin- 9 prevented the induction of IFN- $\gamma$ secretion and also suppressed the production of IL-10 by PBMC. Furthermore, we show that galectin- 9 induces $T_{\text {reg }}$ and $T_{h} 1$ polarization through interaction with antigen-presenting cells. Our findings show that galectin- 9 secreted by IEC apically exposed to TLR9 ligand in the presence of scGOS/lcFOS is involved in $\mathrm{T}_{\mathrm{h}} 1$ and $\mathrm{T}_{\text {reg }}$ polarization and may be a promising target to prevent or treat allergic disease. Copyright $\odot 2013 \mathrm{~S}$. Karger AG, Basel

\section{Introduction}

The gastrointestinal immune system is the largest immunological compartment present in the human body which constantly faces both harmful and harmless antigens present in the intestinal lumen. The intestinal mucosa has therefore the task to discriminate between inducing an immune response in case of pathogen invasion and maintaining tolerance to harmless food antigens or the commensal microbiota. Loss of tolerance towards food antigens may result in the development of food allergy [1]. A monolayer of intestinal epithelial cells (IEC) provides an important barrier between the intestinal lu-

\section{KARGER}

E-Mail karger@karger.com

www.karger.com/jin (c) 2013 S. Karger AG, Basel

$1662-811 \mathrm{X} / 13 / 0056-0625 \$ 38.00 / 0$
Dr. Linette E.M. Willemsen

Division of Pharmacology, Utrecht Institute of Pharmaceutical Sciences Faculty of Science, Utrecht University, PO Box 80082

NL-3508 TB Utrecht (The Netherlands)

E-Mail l.e.m.willemsen@uu.nl 
men and the lamina propria, but it is also known to be involved in the modulation of innate and adaptive immune responses $[2,3]$.

IEC are known to contribute to effector immune responses and have the capacity to express pathogen recognition receptors including Toll-like receptors (TLR). TLR recognize fragments of bacteria for example. Human IEC were found to express TLR at the surface, and TLR expression is increased under inflammatory conditions, whereas under homeostasis IEC are unresponsive toward TLR ligands [4-9]. Epithelial TLR9 ligation by unmethylated bacterial CpG DNA was found to induce tolerance to subsequent TLR stimuli, illustrating its involvement in the maintenance of intestinal homeostasis [10]. In vitro, IEC modulate effector immune responses in a polarized fashion as apical, but not basolateral TLR9 ligand exposure enhances IFN- $\gamma$ and IL-10 secretion, while suppressing IL-13 production by peripheral blood mononuclear cells (PBMC) [11]. In a murine model of peanut allergy, oral administration of TLR9 agonists prevented allergy, linking TLR9 activation to a reduction in allergic diseases [12]. Upon activation, IEC secrete mediators that shape the phenotype of dendritic cells (DC) to induce regulatory $\mathrm{T}$-cell $\left(\mathrm{T}_{\text {reg }}\right)$ differentiation and to suppress the development of both $\mathrm{T}_{\mathrm{h}} 1$ and $\mathrm{T}_{\mathrm{h}} 17$ cells $[3,13]$.

As IEC play an important role in the recognition of microbial stimuli, dietary intervention using specific bacterial strains may be beneficial in modulating effector immune responses in the intestine. Previously, the effects of oral administration of Bifidobacterium breve M-16V, $B$. infantis, B. animalis, Lactobacillus plantarum and $L$. rhamnosus on the suppression of airway responses, pulmonary inflammation, ovalbumin-specific immunoglobulins and cytokines present in serum were assessed in a murine model of asthma. In this study, $B$. breve M-16V was identified as the most potent bacterial strain to suppress allergic responses upon allergen challenge [14]. Follow-up in vitro studies using UV-killed whole bacteria showed that in contrast to Lactobacillus GG, B. breve M$16 \mathrm{~V}$ did not modulate the effector immune response in IEC/PBMC cocultures [15]. Recently, it has been reported that both DNA from commensals as well as probiotic bacterial strains contain immunosuppressive CpG motifs, thereby facilitating $\mathrm{T}_{\text {reg }}$ conversion and anti-inflammatory cytokine production $[16,17]$. Hence, DNA derived from $B$. breve $\mathrm{M}-16 \mathrm{~V}$, but not membrane components, may play a role in modulating effector immune responses through activation of TLR9 on IEC. In addition, a combination of $B$. breve $\mathrm{M}-16 \mathrm{~V}$ with a specific prebiotic 9:1 mixture of short-chain galacto-oligosaccha- rides (scGOS; [Gal $\beta 1-4]_{3-8} \mathrm{Glc}$; Gal, galactose; Glc, glucose) and long-chain fructo-oligosaccharides (lcFOS, $[\text { Frc } \beta 2-1]_{>20}$ Frc $\beta 2-1 G l c ;$ Frc, fructose) (scGOS/lcFOS) most effectively protects against the development of allergic symptoms in a murine model of cow's milk allergy as well as in infants at risk $[18,19]$. Although human milk nondigestible oligosaccharides as well as scGOS/lcFOS are able to shape the intestinal microflora and may induce mucosal tolerance $[20,21]$, it is not known whether these oligosaccharides directly contribute to the modulation of effector immune responses in the intestine.

Receptors involved in the recognition of carbohydrate structures, lectins, may be involved in the recognition of nondigestible oligosaccharides. One family of solubletype lectins expressed by IEC that contain carbohydrate recognition domains are galectins, which exhibit binding specificity for $\beta$-galactosides [22, 23]. IEC were found to express galectin-2, $-3,-4$ and $-9[24,25]$. Galectins are localized in the cytoplasm, but can also be secreted through yet unknown mechanisms. Upon secretion, galectins can bind to glycosylated proteins thus forming galectin-glycoprotein lattices on the cell surface, thereby regulating immune responses and potentially inducing immunological tolerance $[26,27]$. We have previously shown that dietary intervention using scGOS/lcFOS in combination with $B$. breve $\mathrm{M}-16 \mathrm{~V}$ enhances serum galectin-9 levels. To this end, we studied the effects of IEC exposure to scGOS/ lcFOS and TLR9 ligand on human PBMC in an in vitro coculture system [11]. We demonstrate that galectin-9 is expressed and secreted by IEC upon apical exposure to TLR9 ligand and scGOS/lcFOS, which drives a $T_{h} 1 / T_{\text {reg }}$ response.

\section{Materials and Methods}

\section{Transwell Cocultures}

Culture of human IEC lines (HT-29 and T84 cells), isolation of human PBMC and transwell cocultures were performed as previously described [11]. In short, HT-29 or T84 cells were grown till confluence on transwell insert filters (Corning, N.Y., USA). Confluence was examined by light microscopy or transepithelial electrical resistance $\left(125 \Omega \times \mathrm{cm}^{2}\right.$ for HT-29 cells and $1,500 \Omega \times \mathrm{cm}^{2}$ for polarized T84 cell monolayers). IEC were cocultured with $3 \times$ $10^{6} \mathrm{CD} 3 / \mathrm{CD} 28$-activated PBMC for $24 \mathrm{~h}$. IEC were apically exposed to either TLR9 ligand (M362 type C, $5.0 \mu \mathrm{M}$; InvivoGen, San Diego, Calif., USA) alone or in combination with $0.5 \% \mathrm{w} / \mathrm{v}$ of a 9: 1 mixture of scGOS (Vivinal GOS; Borculo Domo) and lcFOS (Raftiline HP; Orafti). For the determination of galectin secretion by IEC, HT-29 cells were placed on fresh medium for another $24 \mathrm{~h}$ after coculture with CD3/CD28-activated PBMC. To study the involvement of galectins in immune modulation, lactose (100 mM; Sigma, Zwijndrecht, The Netherlands) was added to the ba- 
solateral compartment during coculture. Sucrose (100 mM; Sigma) was used as negative control. To specifically block galectin-9, a TIM-3-Fc fusion protein $(1.0 \mu \mathrm{g} / \mathrm{ml}$; R\&D Systems, Minneapolis, Minn., USA) was added to IEC/PBMC cocultures. In addition, HT-29 cells were basolaterally exposed to medium containing recombinant TNF- $\alpha$ and IFN- $\gamma$ (both $10 \mathrm{ng} / \mathrm{ml}$; Invitrogen, Carlsbad, Calif., USA) to study whether inflammatory conditions are essential for HT-29 cells to produce galectin-9 upon apical exposure to TLR9 ligand and scGOS/lcFOS.

\section{Generation of Monocyte-Derived DC}

CD14+ monocytes were isolated from PBMC by negative selection using MACS beads (monocyte isolation kit II; Miltenyi Biotec, Bergisch Gladbach, Germany) and cultured in RPMI 1640 supplemented with $10 \%$ FCS and penicillin $(100 \mathrm{U} / \mathrm{ml}) /$ streptomycin [100 $\mu \mathrm{g} / \mathrm{ml}$; monocyte-derived DC (moDC) medium] with IL-4 and GM-CSF (both Prospec, East Brunswick, N.J., USA) at a concentration of 30 and $15 \mathrm{ng} / \mathrm{ml}$, respectively, and incubated for 6 days at $37^{\circ} \mathrm{C}$ and $5 \% \mathrm{CO}_{2}$. After 2, 3 and 6 days, $1 \mathrm{ml}$ medium was refreshed. For moDC conditioning experiments, monocytes were differentiated into moDC with IL- 4 and GM-CSF in the presence of recombinant human galectin- $9(1.0 \mu \mathrm{g} / \mathrm{ml}$; R\&D Systems $)$. On day 7 , the phenotype of moDC was confirmed by FACS.

\section{In vitro T-Cell Differentiation}

In mixed lymphocyte cultures, $2 \times 10^{4} \mathrm{moDC}$ were seeded per well and cultured with naive allogeneic CD4+ T cells $\left(10^{5}\right)$. For

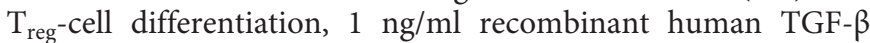
(Prospec) was added to the cultures. Cultures were incubated at $37^{\circ} \mathrm{C}$ and $5 \% \mathrm{CO}_{2}$ for 5 days. Before FACS analysis of intracellular cytokines, cells were re-stimulated with phorbol-12-myristate13 -acetate (50 ng/ml; Sigma) and ionomycin (750 ng/ml; Sigma) for $6 \mathrm{~h}$. After $1 \mathrm{~h}$ of restimulation, GolgiPlug (BD Biosciences, San Jose, Calif., USA) was added according to the manufacturer's protocol. CD $4+$ cells producing IL-10 and IFN- $\gamma$ were analyzed by flow cytometry.

Culture and Isolation of Genomic DNA of B. breve M-16V

$B$. breve M-16V was cultivated in Mann Rogosa Sharp broth (Oxoid) for $48 \mathrm{~h}$ at $37^{\circ} \mathrm{C}$ under anaerobic conditions. Genomic DNA was extracted and purified by CTAB extraction as described [28] with additional lysozyme treatment of the cell suspension $\left(2 \mathrm{mg} / \mathrm{ml}, 5 \mathrm{~min}\right.$ at $\left.20^{\circ} \mathrm{C}\right)$.

\section{ELISA}

Concentrations of IL-6, IL-10, IL-12, IFN- $\gamma$, TNF- $\alpha$ (CytoSets ${ }^{\mathrm{TM}}$; Biosource, Nivelles, Belgium), IL-13 (R\&D Systems) and IL-17A (Arcus Biologicals, Modena, Italy) were measured according to the manufacturer's protocol. For galectin- 4 and galectin- 9 ELISA, high-binding EIA/RIA 96-well plates (Costar; Corning Inc., Corning, N.Y., USA) were coated with $0.75 \mu \mathrm{g} / \mathrm{ml}$ primary antibodies (R\&D Systems) dissolved in PBS overnight at $4{ }^{\circ} \mathrm{C}$. Plates were blocked for $1 \mathrm{~h}$ with $1 \%$ BSA in PBS, samples were then added for $2 \mathrm{~h}$, followed by incubation with $0.75 \mu \mathrm{g} / \mathrm{ml}$ biotinylated secondary antibody (R\&D Systems) in 1\% BSA in PBS for $1 \mathrm{~h}$. Plates were incubated with streptavidin-HRP (R\&D Systems) for $1 \mathrm{~h}$ followed by development with tetramethylbenzidine (Thermo Scientific, Rockford, Ill., USA) for $10 \mathrm{~min}$. The reaction was stopped with $2 \mathrm{M} \mathrm{H}_{2} \mathrm{SO}_{4}$ and optical density was measured at 450 $\mathrm{nm}[29]$.

scGOS/lcFOS Supports $\mathrm{T}_{\mathrm{h}} 1 / \mathrm{T}_{\text {reg }}$

Responses via Galectin-9

\section{Flow Cytometry}

$\mathrm{T}_{\mathrm{h}} 1$ cells were stained with CD4-PE-Cy5 (eBioscience, San Diego, Calif., USA), CD69-PE (eBioscience) and CXCR3-Alexa Fluor 488 (BD Biosciences) and fixed with $0.5 \%$ paraformaldehyde. $\mathrm{T}_{\text {reg }}$ cells were stained with CD4-PE-Cy5 and CD25-Alexa Fluor 488 (eBioscience), followed by intracellular staining with Foxp3$\mathrm{PE}$ using the Foxp3 staining set according to manufacturer's protocol (eBioscience). For intracellular cytokine staining, PBMC were restimulated with phorbol-12-myristate-13-acetate (50 ng/ $\mathrm{ml}$; Sigma) and ionomycin (750 ng/ml; Sigma) for $6 \mathrm{~h}$ in the presence of a Golgi transport inhibitor according to the manufacturer's instructions (GolgiPlug) followed by intracellular staining using IFN- $\gamma$-antigen-presenting cells (APC) and IL-10-PE (BD Biosciences) as described above. Flow-cytometric analysis was performed using a FACSCanto II (BD Biosciences).

\section{cDNA Synthesis and Real-Time PCR}

HT-29 cells were washed once in PBS $2 \mathrm{~h}$ after coculture with CD3/CD28-activated PBMC and taken up in $200 \mu \mathrm{l}$ RAAlater ${ }^{\mathrm{TM}}$ (Qiagen GmbH, Hilden, Germany). Samples were stored at $-20^{\circ} \mathrm{C}$ until cDNA synthesis. mRNA was isolated using the mRNA capture kit (Roche, Mannheim, Germany) and real-time PCR reactions were performed as previously described [30]. GAPDH was used as reference gene. Relative target mRNA abundance was calculated by applying the formula: relative mRNA abundance $=100$ $\times 2^{\mathrm{Ct}[G A P D H]-\mathrm{Ct}[\operatorname{target} \mathrm{mRNA}]}$. Primers for all galectins were designed using the computer software Primer Express 2.0 (Applied Biosystems, Carlsbad, Calif., USA).

\section{Fluorescence Microscopy Staining of Galectin-9 in IEC}

IEC were fixed using $4 \%$ formalin in PBS for $10 \mathrm{~min}$, permeabilized in $0.1 \%$ Triton X-100 (Sigma) and 1\% BSA in PBS for 15 min and incubated with anti-human galectin- 4 or galectin- 9 antibodies or normal goat IgG as isotype control (all $0.75 \mu \mathrm{g} / \mathrm{ml}$; R\&D Systems) in $0.1 \%$ Triton X-100 and $1 \%$ BSA in PBS for $1 \mathrm{~h}$. IEC were incubated with secondary Alexa Fluor 546 donkey anti-goat IgG (Invitrogen) in $0.1 \%$ Triton X-100 and $1 \%$ BSA for $30 \mathrm{~min}$ and embedded in Hoechst. Object glasses were stored at $4^{\circ} \mathrm{C}$ until microscopic examination.

\section{Statistics}

Statistical analyses were performed using paired Student's t test or one-way ANOVA for repeated measurements followed by Bonferroni's post hoc test. Analyses were performed using GraphPad Prism 5.0. $\mathrm{p}<0.05$ was considered statistically significant.

\section{Results}

\section{The scGOS/lcFOS Mixture Specifically Enhances a}

TLR9-Induced IFN- $\gamma$ Response via IEC

To investigate the molecular mechanisms by which scGOS/lcFOS exerts its immunomodulatory effects, we used an in vitro coculture model with human IEC and PBMC to study IEC-immune cell crosstalk. We hypothesized that DNA of B. breve M-16V may activate TLR9 on 
IEC as apical TLR9 ligation of IEC can induce a regulatory-type $T_{h} 1$ response [11]. IEC apically exposed to purified DNA from B. breve M-16V or a synthetic TLR9 ligand enhanced IFN- $\gamma$ secretion by activated PBMC, which was potentiated by scGOS/lcFOS (fig. 1a). Direct stimulation of CD3/CD28-activated PBMC with $\mathrm{CpG}$ DNA in the presence of scGOS/lcFOS did not enhance IFN- $\gamma$ secretion (fig. 1d). Furthermore, it was observed that $s c G O S / l c F O S$ did not increase IFN- $\gamma$ production on their own, indicating that the presence of both scGOS/ lcFOS and the bacterial DNA is essential for the observed additional effect. In addition, both in the presence and absence of IEC, the production of the $\mathrm{T}_{\mathrm{h}} 2$-associated cytokine IL-13 was significantly reduced upon apical exposure of HT-29 to synthetic CpG DNA. The presence of scGOS/lcFOS did not modulate IL-13 secretion by PBMC in the presence of HT-29 cells; in the absence of IEC IL13 secretion was reduced. The combination of both $\mathrm{CpG}$ DNA and scGOS/lcFOS suppressed IL-13 in the presence and absence of HT-29 cells. However, exposure of HT-29 cells to the genomic DNA derived from $B$. breve M-16V did not result in decreased IL-13 secretion in the presence or absence of scGOS/lcFOS (fig. 1b, e).

In addition to the induction of $\mathrm{T}_{\mathrm{h}} 1$-polarized effector response, we studied whether IEC apically exposed to TLR9 ligand in the presence of scGOS/lcFOS enhances anti-inflammatory IL-10 production as well. TLR9 ligation on IEC, as well as exposure of IEC to DNA from $B$. breve $\mathrm{M}-16 \mathrm{~V}$, resulted in increased IL-10 secretion. TLR9-induced IL-10 secretion by CD3/CD28-activated PBMC was not affected by scGOS/lcFOS (fig. 1c). In the absence of IEC, the TLR9 ligand enhanced IL-10 secretion as well, and a similar tendency was observed in the presence of scGOS/lcFOS (fig. 1f).

\section{$T_{h} 1$ and $T_{\text {reg }}$ Cells Are Increased by IEC Exposed to}

TLR9 Ligand in the Presence of scGOS/lcFOS

To confirm whether scGOS/lcFOS in combination with CpG DNA modulates T-cell polarization, T-cell phenotype was assessed. $T_{h} 1$ cells characterized as either CD4+CD69+CXCR3+ cells and IFN- $\gamma$-expressing CD4+ $\mathrm{T}$ cells were analyzed. An increased frequency of CD4+CD69+CXCR3+ $\mathrm{T}_{\mathrm{h}} 1$ cells was observed when IEC were apically exposed to TLR9 ligand, which was further increased by scGOS/lcFOS (fig. 2a, b). In the absence of IEC, the percentage of activated $\mathrm{T}_{\mathrm{h}} 1$ cells remained unaltered (fig. 2c). To confirm the induction of $\mathrm{T}_{\mathrm{h}} 1$ cells, intracellular IFN- $\gamma$ staining was performed. Similarly, only in the presence of IEC, the percentage of IFN- $\gamma$-producing CD4+ T cells was increased upon apical exposure of IEC to TLR9 ligand, which was boosted by scGOS/lcFOS (fig. 2d, e).

Since CpG DNA and genomic DNA derived from $B$. breve $\mathrm{M}-16 \mathrm{~V}$ enhanced anti-inflammatory IL-10 secretion, the frequency of CD4+CD25+Foxp3+ $\mathrm{T}_{\text {reg }}$ cells was analyzed, too. The percentage of $\mathrm{T}_{\text {reg }}$ cells was only increased when IEC were apically exposed to TLR9 ligand in the presence of scGOS/lcFOS $(7.7 \pm 1.2 \%$ for mediumexposed IEC vs. $10.4 \pm 1.5 \%$ for IEC exposed to CpG DNA in the presence of scGOS/lcFOS; fig. 3a-c). Likewise, intracellular IL-10 expression by $\mathrm{CD} 4+\mathrm{T}$ cells was increased upon TLR9 ligation in the presence and absence of IEC, however, scGOS/lcFOS did not enhance IL-10 production by $\mathrm{CD} 4+\mathrm{T}$ cells (fig. $3 \mathrm{~d}, \mathrm{e}$ ).

\section{Galectin-9 Expression by IEC Is Increased upon}

Exposure to TLR9 Ligand and scGOS/lcFOS

Galectins expressed by IEC may be involved in the induction of $\mathrm{T}_{\mathrm{h}} 1$ - and $\mathrm{T}_{\text {reg-cell development. To address }}$ this possibility, we evaluated whether apical exposure of HT-29 cells to TLR9 ligand and scGOS/lcFOS modulates epithelial galectin expression. First, it was assessed which galectins are expressed by HT-29 cells when cocultured with CD3/CD28-activated PBMC (fig. 4a). Galectin-4 and the long isoform of galectin-9 were highly expressed by HT-29 cells. Apical TLR9 ligation of IEC increased galectin-9 mRNA expression by HT-29, but not galectin-4, while in the presence of scGOS/lcFOS galectin-9 expression was further enhanced (fig. 4b, c). Furthermore, enhanced basolateral secretion of galectin- 9 was observed upon epithelial exposure to TLR9 ligand, which was significantly potentiated by the combination with scGOS/ lcFOS (fig. 4d). Galectin-4 expression by HT-29 cells was not modulated upon apical exposure of HT-29 cells to TLR9 ligand or scGOS/lcFOS (fig. 4c, f). Hence, TLR9 ligand and scGOS/lcFOS specifically enhanced galectin-9 expression and secretion by IEC.

In addition, galectin- 9 secretion by HT-29 upon apical exposure to TLR9 ligand in the presence of scGOS/lcFOS did not occur under noninflammatory conditions. Unstimulated HT-29 cells secreted low levels of galectin-9 (55.6 \pm 3.7 vs. $56.9 \pm 10.3 \mathrm{pg} / \mathrm{ml}$ for medium vs. TLR9 ligand and scGOS/lcFOS-exposed HT-29, n = 4). However, exposure of pre-incubated HT-29 cells with proinflammatory cytokines TNF- $\alpha$ and IFN- $\gamma$ to TLR9 ligand and scGOS/lcFOS resulted in enhanced galectin-9 secretion (114.0 \pm 43.6 vs. $246.0 \pm 36.6 \mathrm{pg} / \mathrm{ml}$ for medium vs. TLR9 ligand and scGOS/ lcFOS-exposed HT-29, $\mathrm{n}=4, \mathrm{p}<0.05)$. These data indicate that IEC respond to TLR9 ligand in the presence of scGOS/ lcFOS only under inflammatory conditions. 

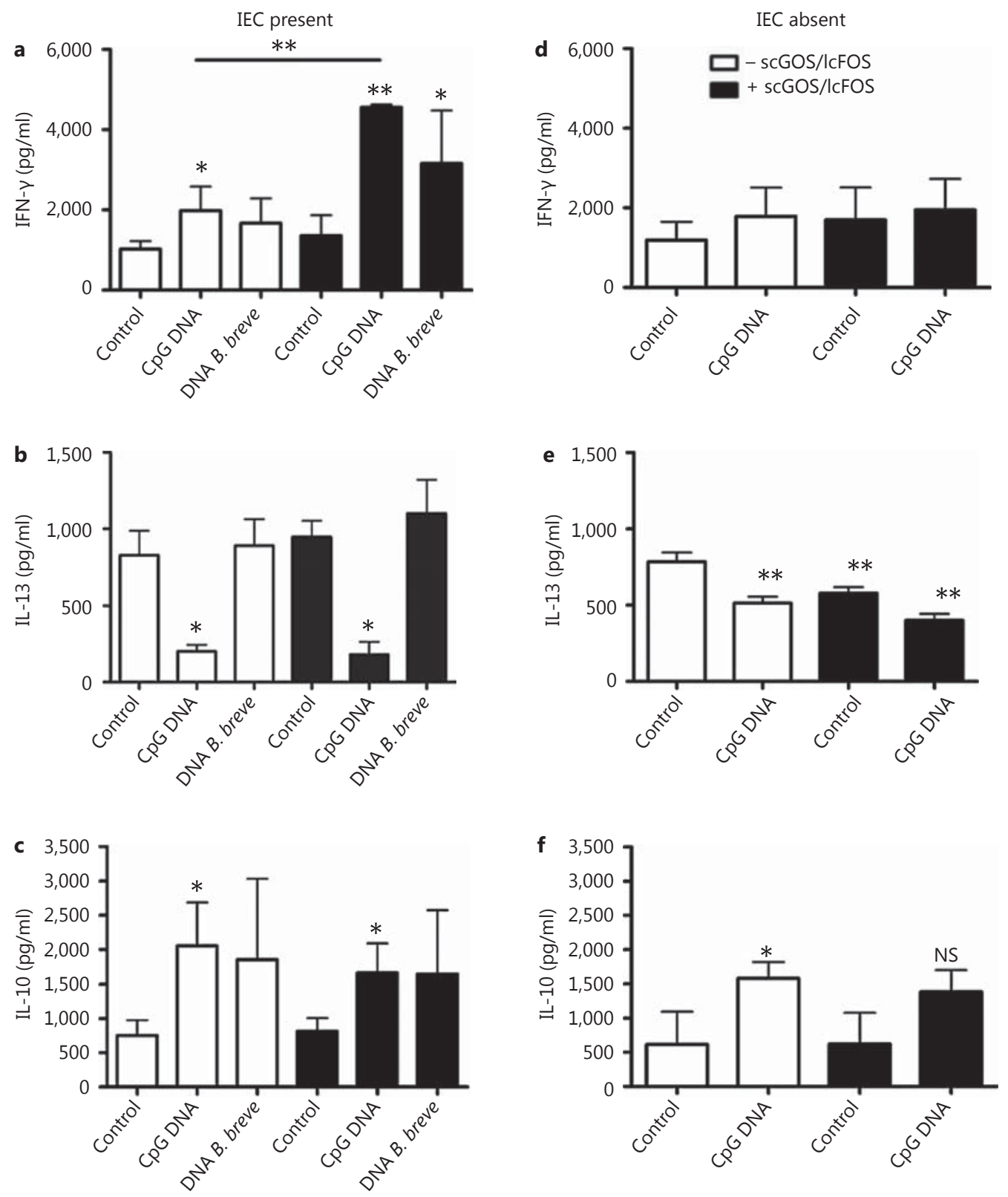

Fig. 1. TLR9 ligation of IEC in the presence of scGOS/lcFOS modulates cytokine secretion by PBMC. HT-29 cells were apically exposed to TLR9 ligand or DNA from $B$. breve M-16V in the presence or absence of scGOS/lcFOS and cocultured for $24 \mathrm{~h}$ with CD3/CD28-activated PBMC. In addition, PBMC were directly stimulated with TLR9 ligand in the presence of scGOS/lcFOS. Exposure of HT-29 cells to TLR9 ligand or DNA from B. breve $\mathrm{M}-16 \mathrm{~V}$ increased IFN- $\gamma$ secretion by PBMC, which was further enhanced in the presence of scGOS/lcFOS (a). This was not ob- served in the absence of HT-29 cells (d). The secretion of IL-10 was enhanced upon apical exposure of HT-29 cells to TLR9 ligand or DNA from $B$. breve M-16V, while IL-13 production was reduced. The presence of scGOS/lcFOS did not affect IL-13 (b) and IL-10 secretion (c). The effects on IL-10 and IL-13 secretion by PBMC were also observed in the absence of HT-29 cells $(\mathbf{e}, \mathbf{f})$. Means \pm SEM of 3-4 independent PBMC donors. ${ }^{*} \mathrm{p}<0.05$, ${ }^{* *} \mathrm{p}<0.01,{ }^{* * *} \mathrm{p}<0.001$. 


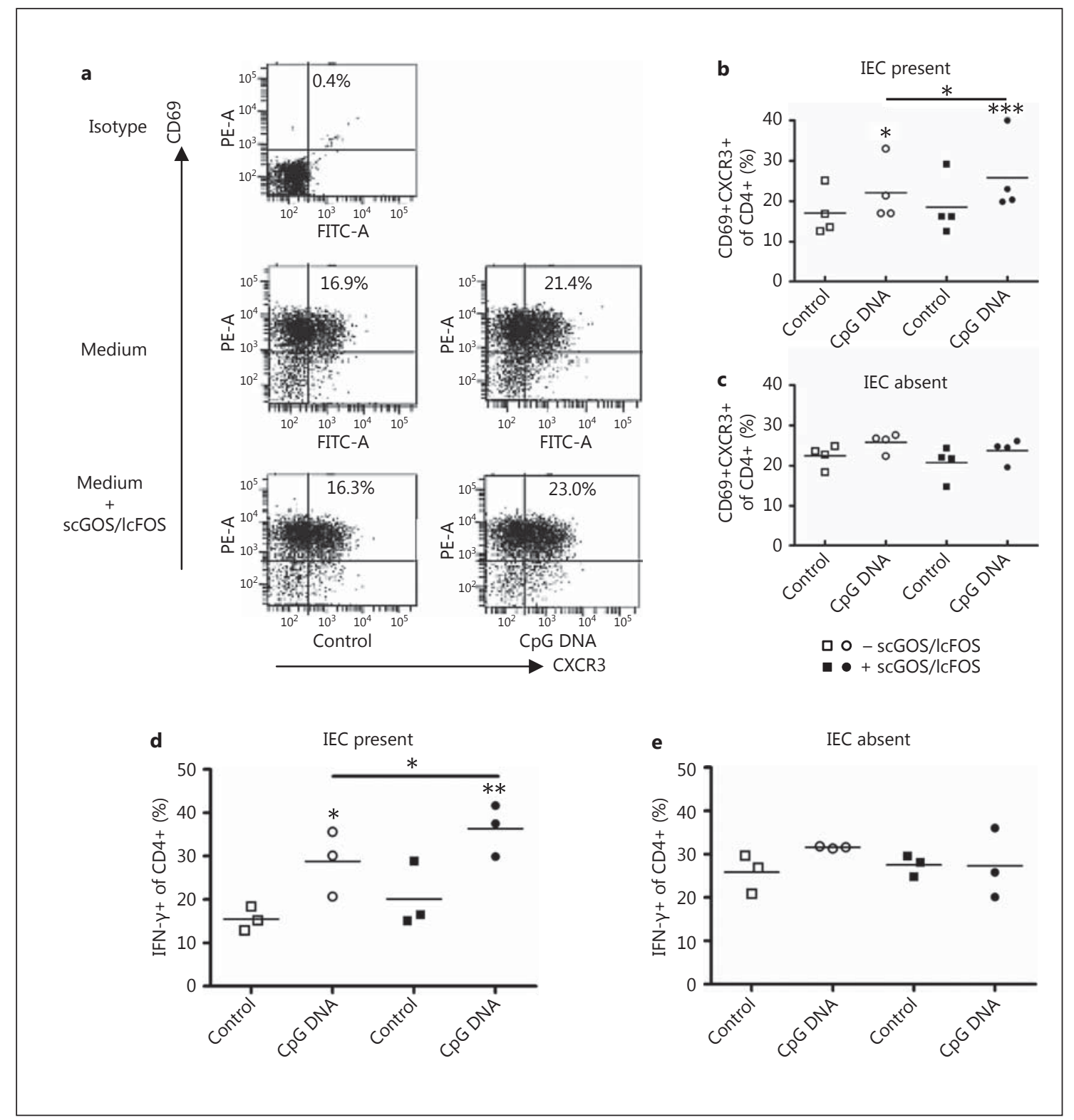

Fig. 2. TLR9 ligation of IEC in the presence of scGOS/lcFOS results in an enhanced $\mathrm{T}_{\mathrm{h}} 1$-type effector response. HT-29 cells were apically exposed to TLR9 ligand in the presence or absence of scGOS/ lcFOS and cocultured for $24 \mathrm{~h}$ with CD3/CD28-activated PBMC. In addition, PBMC were directly stimulated with TLR9 ligand in the presence of scGOS/lcFOS. The frequency of activated $\mathrm{T}_{\mathrm{h}} 1$ cells $(\mathrm{CD} 4+\mathrm{CD} 69+\mathrm{CXCR} 3+)$ and $\mathrm{CD} 4+\mathrm{IFN}-\gamma+$ cells was only in- creased by apical TLR9 ligation of IEC, which was further enhanced by the presence of scGOS/lcFOS $(\mathbf{a}, \mathbf{b}, \mathbf{d})$. Direct stimulation of PBMC with TLR9 ligand in the absence or presence of scGOS/lcFOS did not alter the percentage of $\mathrm{T}_{\mathrm{h}} 1$ cells $(\mathbf{c}, \mathbf{e})$. Means \pm SEM of 3-4 independent PBMC donors. ${ }^{*} \mathrm{p}<0.05,{ }^{* *} \mathrm{p}<0.01$, $* * * \mathrm{p}<0.001$.
IEC-Derived Galectin-9 Secretion Is Involved in the Induction of IFN- $\gamma$ and IL-10 Polarized-Type Effector Responses

In order to study the involvement of galectins in the induction of $\mathrm{T}_{h} 1$ and $\mathrm{T}_{\text {reg }}$ differentiation, galectin func- tion was inhibited by the addition of lactose to the basolateral compartment of HT-29/PBMC cocultures. Lactose binds galectins through their carbohydrate recognition domain and thereby prevents binding of galectins to glycan structures expressed on the cell surface. To deter- 


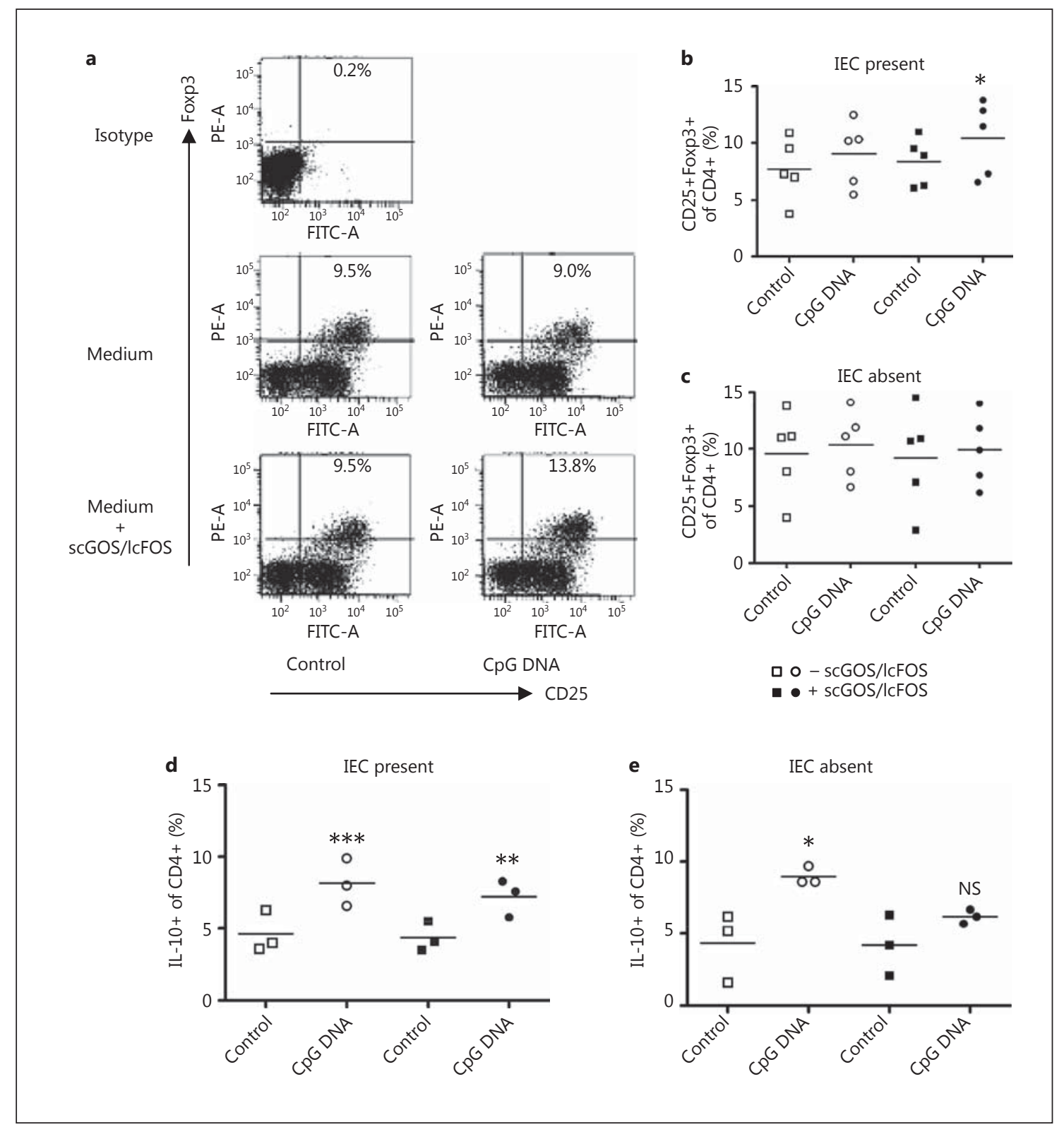

Fig. 3. TLR9 ligation of IEC in the presence of scGOS/lcFOS results

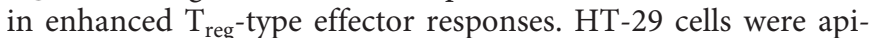
cally exposed to TLR9 ligand in the presence or absence of scGOS/ lcFOS and cocultured for $24 \mathrm{~h}$ with CD3/CD28-activated PBMC. In addition, PBMC were directly stimulated with TLR9 ligand in presence of scGOS/lcFOS. The frequency of $\mathrm{T}_{\text {reg }}$ cells (CD4+CD25+Foxp3+) was only increased by apical TLR9 ligation of IEC in the presence of scGOS/lcFOS $(\mathbf{a}, \mathbf{b})$. Direct stimulation of PBMC with TLR9 ligand in the absence or presence of scGOS/ lcFOS did not modulate the number of $\mathrm{T}_{\text {reg }}$ cells $(\mathbf{c})$. Intracellular IL-10 expression by CD4+ T cells was increased by TLR9 ligand and remained unaltered in the presence of scGOS/lcFOS (d). Similar results were observed in the absence of IEC (e). Means \pm SEM of 3-5 independent PBMC donors, ${ }^{*} \mathrm{p}<0.05$. mine the specificity of the intervention, sucrose was used as a negative control. Neutralization of galectins by lactose in the basolateral compartment in HT-29/PBMC cocultures exposed to TLR9 ligand and scGOS/lcFOS resulted in decreased IFN- $\gamma$ and IL-10 secretion (fig. 5a, b), while the production of IL-13 was increased (fig. $5 c$ ). In addition, IL-6, IL-12, IL-17 and TNF- $\alpha$ secretion were increased by adding lactose to the basolateral compartment during coculture (fig. $5 \mathrm{~d}-\mathrm{g}$ ). Thus, inhibition of galectins induced by IEC exposed to TLR9 ligand and scGOS/ 


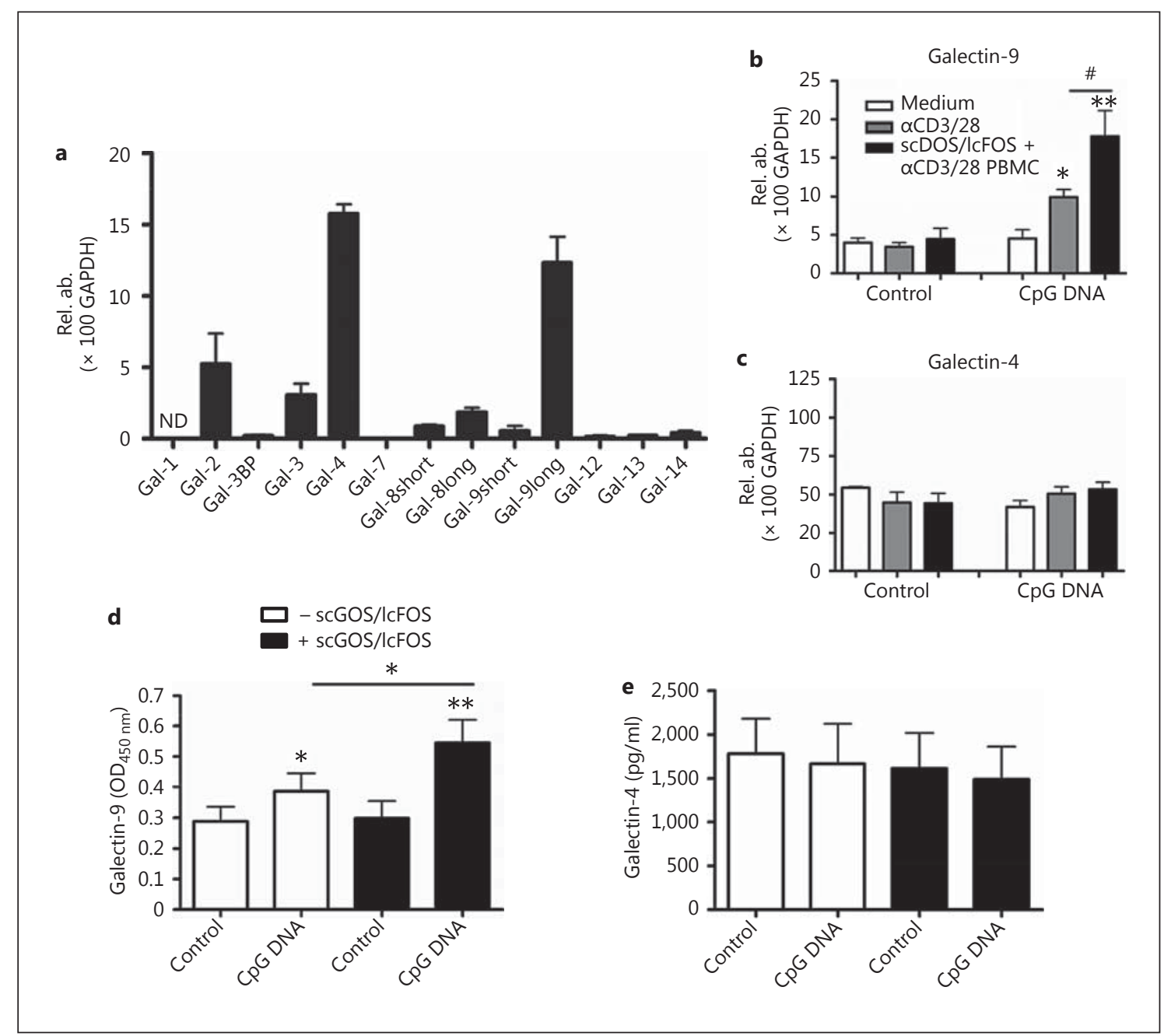

Fig. 4. Expression and secretion of galectin-9 in HT-29 cells is regulated by TLR9 ligation and scGOS/lcFOS. a-c Analysis of galectin (Gal) mRNA expression by HT-29 cells by quantitative PCR analysis. Evaluation of the expression profile of galectins by IEC (a). Apical TLR9 ligation of HT-29 cells, in the absence or presence of scGOS/lcFOS, specifically increases galectin-9 expression (b, c). Protein expression of galectin- 4 and galectin- 9 and its modulation upon TLR9 ligation and scGOS/lcFOS was confirmed by immunofluorescence microscopic staining of HT-29 monolayers (d). ELISA was performed in the basolateral supernatant of HT-29 monolayers (e). TLR9 ligation of HT-29 cells in the presence of scGOS/lcFOS enhanced galectin-9, but not galectin-4 secretion. $\mathrm{n}=3(\mathbf{a}-\mathbf{d})$ or $\mathrm{n}=6(\mathbf{e})$ independent PBMC donors, means \pm SEM, ${ }^{*} \mathrm{p}<0.05,{ }^{*} \mathrm{p}<0.05,{ }^{* *} \mathrm{p}<0.01$.
lcFOS abrogated the induction of IFN- $\gamma$ and IL-10 as well as the reduction in IL-13 secretion by PBMC upon apical exposure of IEC to TLR9 ligand in the presence of scGOS/ lcFOS. In contrast, galectin neutralization enhanced a proinflammatory response.

To prove that galectin- 9 is involved in inducing IFN- $\gamma$ and IL-10-mediated effector responses, a TIM-3-Fc fusion protein was used to specifically neutralize secreted galectin-9. TIM-3-Fc prevented increased IFN- $\gamma$ and IL-10 secretion by activated PBMC in HT-29/PBMC cocultures, but did not modulate IL-17 production (fig. 6a-c). Similar results were obtained using the polarized T84 cell line (fig. 6e-g). These data collectively suggest that IEC contribute to enhanced IFN- $\gamma$ - and IL-10-type effector responses upon apical exposure to TLR9 ligand and scGOS/ lcFOS through secretion of galectin-9. Apical exposure of HT-29 as well as T84 cells to CpG DNA in the presence of scGOS/lcFOS resulted in suppression of IL-13 secretion. Furthermore, neutralization of galectin-9 abrogated the immunomodulating effect of apical epithelial exposure to 


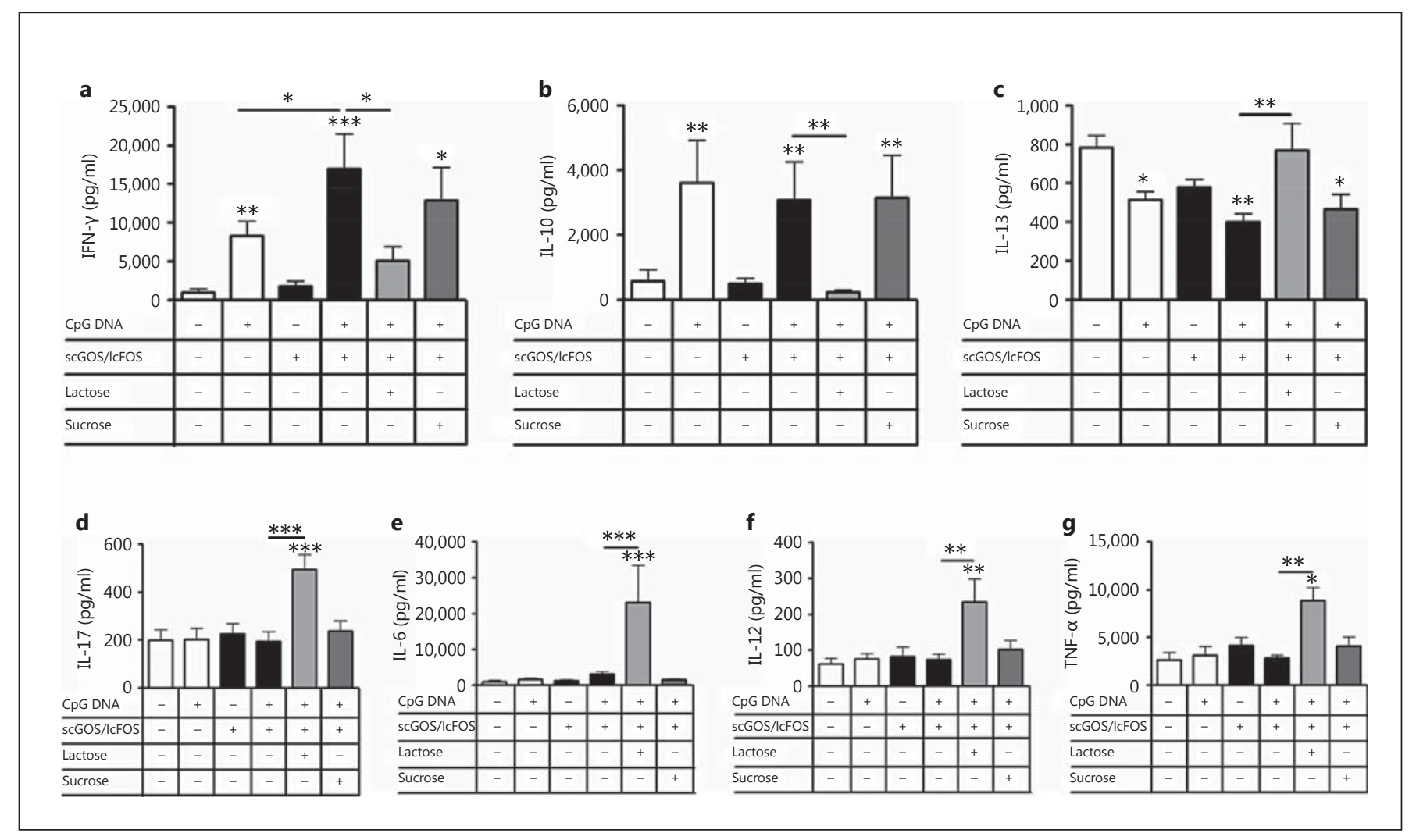

Fig. 5. Neutralization of galectins by lactose redirects the TLR9 and scGOS/lcFOS-induced IFN- $\gamma /$ IL-10-mediated response to a proinflammatory response. HT-29/PBMC cocultures were performed in the presence of lactose or sucrose in the basolateral compartment. Sucrose served as negative control. Presence of lactose, but not sucrose, suppressed IFN- $\gamma$ and IL-10 secretion induced by
CpG DNA and scGOS/lcFOS, and abrogated the reduction in IL13 production $(\mathbf{a}-\mathbf{c})$. The secretion of IL-17, IL-6, IL-12 and TNF- $\alpha$ was increased upon neutralization of galectins by lactose but not sucrose $(\mathbf{d}-\mathbf{g}) . \mathrm{n}=6$ independent PBMC donors, means \pm SEM, ${ }^{*} \mathrm{p}<0.05,{ }^{* *} \mathrm{p}<0.01,{ }^{* * *} \mathrm{p}<0.001$.
Fig. 6. Galectin-9 neutralization in IEC/PBMC cocultures abrogates IFN- $\gamma$ and IL-10 secretion by PBMC. HT-29 (unpolarized) and T84 (polarized) IEC were cocultured with CD3/CD28-activated PBMC for $24 \mathrm{~h}$ and apically exposed to TLR9 ligand in the presence or absence of scGOS/lcFOS. Basolateral galectin- 9 was neutralized using TIM-3-Fc fusion protein. Galectin-9 neutralization abrogated the induction of IFN- $\gamma(\mathbf{a}, \mathbf{e})$ and IL-10 (b, f) by PBMC, but did not modulate IL-17A secretion by $\operatorname{PBMC}(\mathbf{c}, \mathbf{g})$. Furthermore, the immunomodulating effects on IL-13 secretion induced by exposure of HT-29 or T84 cells to TLR9 ligand in the absence or presence of scGOS/lcFOS was abolished upon neutralization of galectin- 9 (d, h). Means \pm SEM of 3 independent PBMC donors. ${ }^{*} \mathrm{p}<0.05,{ }^{* *} \mathrm{p}<0.01,{ }^{* * *} \mathrm{p}<0.001 . \mathrm{GF}=\mathrm{scGOS} / \mathrm{lcFOS}$.

(For figures see next pages.)
Fig. 7. Functional responses of galectin-9-stimulated naïve CD4+CD45RA+ T cells and moDC. To study whether galectin-9 acts on moDC, $T$ cells or via modulation of the interaction between moDC and T cells, galectin- 9 was added during the differentiation of monocytes to moDC or during coculture of moDC with naïve CD4+ T cells (a). Naïve CD4+CD45RA+ T cells, moDC or moDCT-cell cocultures were exposed to recombinant human galectin-9. Mixed lymphocyte cultures were maintained for 5 days. For $\mathrm{T}_{\text {reg- }}{ }^{-}$ cell differentiation, exogenous TGF- $\beta$ was added to the culture. For intracellular cytokines, $\mathrm{T}$ cells were restimulated as described in the Materials and Methods. Stimulation of naïve CD4+ T cells with galectin-9 did not induce CD25+Foxp3+ $\mathrm{T}_{\text {reg }}$ cells $(\mathbf{b}, \mathbf{d})$, IL$10(\mathbf{b}, \mathbf{e})$ or IFN $-\gamma$ expression by CD4+ T cells $(\mathbf{b}, \mathbf{f})$. However, moDC generated in the presence of galectin-9 (Gal-9 DC) or exogenous galectin-9 added to mixed lymphocyte cultures with unconditioned (control) moDC resulted in increased differentiation of CD25+Foxp3+ $\mathrm{T}_{\text {reg }}$ cells (c, d) and IL-10-expressing CD4+ cells (c, e). Only Gal-9 DC had the capacity to induce IFN- $\gamma$ expression by CD $4+\mathrm{T}$ cells $(\mathbf{c}, \mathbf{f}) . \mathrm{n}=3$ independent PBMC donors. ${ }^{*} \mathrm{p}<0.05$, ** $\mathrm{p}<0.01$ vs. control DC.
scGOS/lcFOS Supports $\mathrm{T}_{\mathrm{h}} 1 / \mathrm{T}_{\text {reg }}$

Responses via Galectin-9
J Innate Immun 2013;5:625-638 DOI: $10.1159 / 000350515$ 


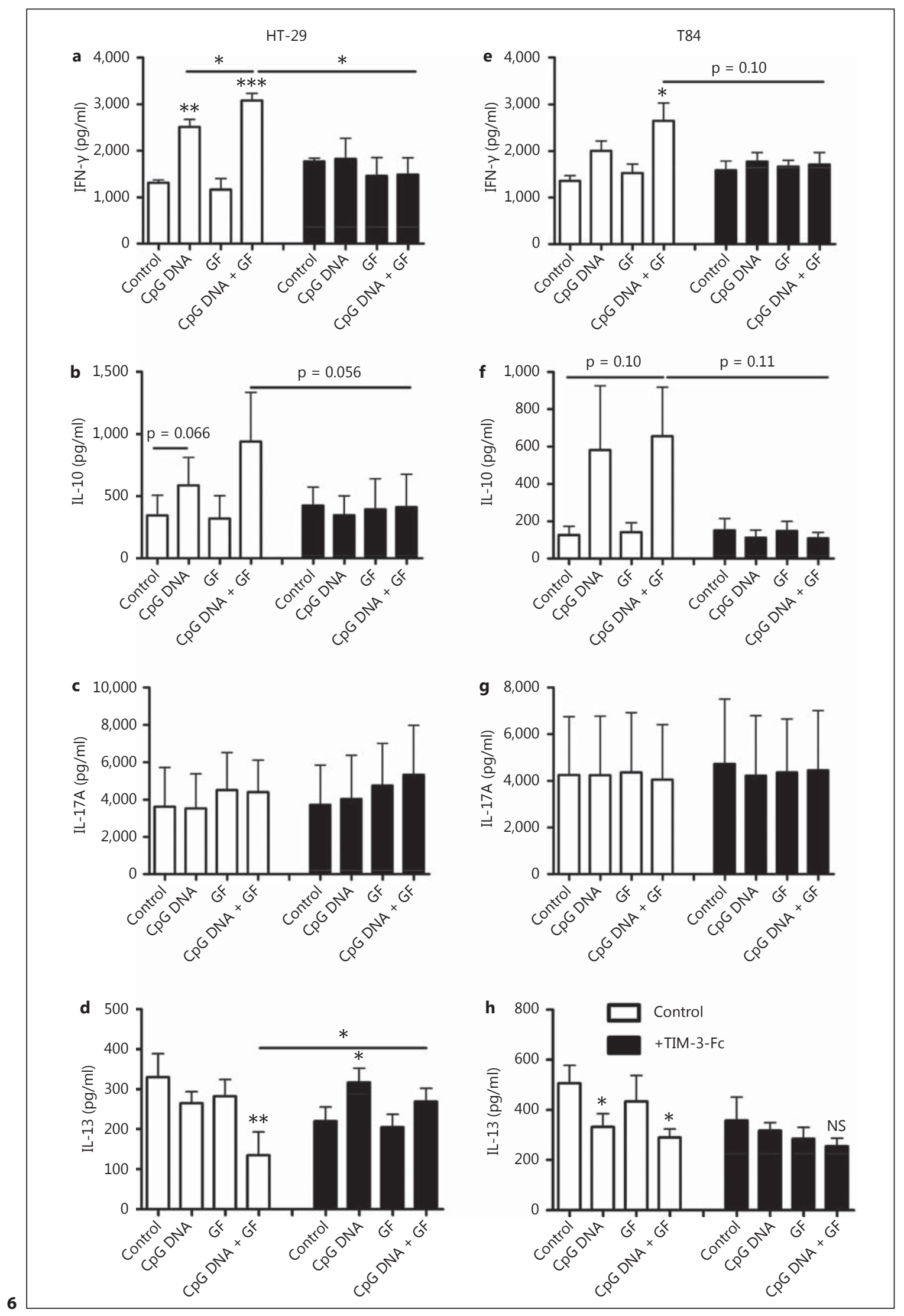




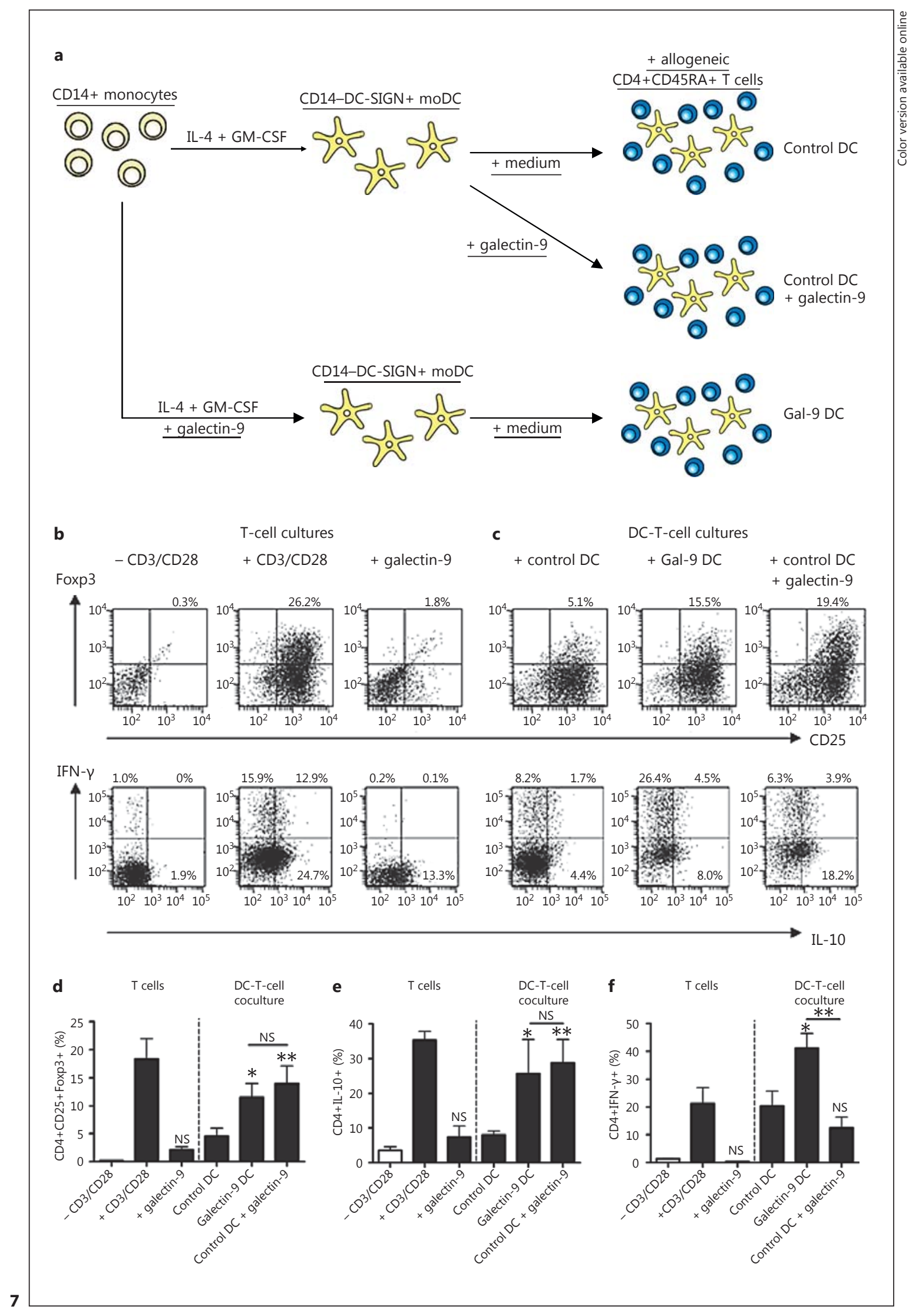


CpG DNA and scGOS/lcFOS regarding IL-13 secretion by PBMC (fig. 6d, h). TIM-3-Fc did not affect the viability of CD3/CD28-activated PBMC (data not shown).

\section{Galectin-9 Induces $T_{\text {reg }}$ and $T_{h} 1$ Polarization through} APC

Finally, we investigated whether galectin- 9 induces $\mathrm{T}$ cell polarization through a direct effect on $\mathrm{T}$ cells or via activation of APC. Under inflammatory conditions, monocytes migrate from the circulation into the intestinal lamina propria [31], where these cells are conditioned by mediators present in the mucosal microenvironment. Therefore, moDC were generated in the absence or presence of galectin-9 prior to mixed lymphocyte cultures. In addition, galectin-9 was added to CD4+CD45RA+ naïve T cells. To evaluate whether galectin-9 plays a role in the interaction between APC and T cells, moDC were cocultured with naïve $\mathrm{CD} 4+\mathrm{T}$ cells in mixed lymphocyte cultures in the presence of galectin-9 (fig. 7a). Exposure of naïve CD4+CD45RA+ T cells to galectin-9 did not induce $\mathrm{T}_{\text {reg }}$ development or cytokine expression (fig. $7 \mathrm{~b}, \mathrm{~d}-\mathrm{f}$ ). However, exposure of moDC to galectin- 9 prior to interaction with naïve $\mathrm{T}$ cells enhanced $\mathrm{T}_{\text {reg }}$ development compared to unconditioned moDC. When galectin-9 was added to moDC-T-cell cultures using control DC, $\mathrm{T}_{\text {reg }}$ polarization was also enhanced (fig. 7c, d). In addition to induction of $\mathrm{T}_{\text {reg }}$ cells, galectin-9-conditioned moDC increased IL-10 and IFN- $\gamma$ production by CD4+ T cells (fig. $7 \mathrm{c}, \mathrm{e}, \mathrm{f}$ ). These data indicate that galectin- 9 conditions APC that have increased capacity to induce $\mathrm{T}_{\text {reg }}$ cells as well as IFN- $\gamma$ - and IL-10-mediated effector responses in vitro.

\section{Discussion}

The intestinal mucosa is constantly facing a high load of antigens derived from the diet and microorganisms for example. The intestinal epithelium serves as the first line of defense against foreign antigens. A single monolayer of IEC forms a tight barrier between the intestinal lumen in the lamina propria. In addition, IEC have been shown to condition DC to induce $\mathrm{T}_{\text {reg }}$ cells [13], indicating that besides providing a barrier, IEC take actively part in innate immune responses and shape adaptive immunity. As IEC are at the interface between the intestinal lumen and the lamina propria, IEC may serve as an interesting target for intervention strategies to modulate immune responses in the gut.

Dietary intervention using $B$. breve $\mathrm{M}-16 \mathrm{~V}$ and scGOS/ lcFOS has been shown to prevent the development of acute allergic symptoms [18]. However, the underlying mechanisms are not yet known. Data presented in this study indicate that the genomic DNA from $B$. breve $\mathrm{M}$ $16 \mathrm{~V}$ enhances IFN- $\gamma$ and IL-10 responses in an IEC-dependent manner. Recently, it was described that oral administration of DNA from $B$. breve enhances IL-10 and TGF- $\beta$ production in the colon, indicating an anti-inflammatory role of $B$. breve DNA [17]. We elaborate on these data by showing that the anti-inflammatory role of $B$. breve $\mathrm{M}-16 \mathrm{~V}$ is similar to the effects exerted by a synthetic CpG DNA. However, the synthetic CpG DNA but not $B$. breve M-16V DNA was capable of suppressing IL13 as well. This may relate to intrinsic immunomodulatory capacities of synthetic CpG DNA, however we cannot exclude the possibility that the purity of the bacterial DNA was suboptimal [17]. Although the combination of synthetic CpG DNA in the presence of scGOS/lcFOS did not consistently further decrease the $\mathrm{T}_{\mathrm{h}} 2$ response, the overall balance of the effector response is in favor of a $\mathrm{T}_{\mathrm{h}} 1$ - and $\mathrm{T}_{\text {reg }}$-polarized response. Furthermore, we have identified galectin- 9 as a soluble mediator released by IEC upon apical TLR9 ligation, which supports IFN- $\gamma$ - and IL-10-polarized effector responses. Regulation of galectin expression is largely unknown, but the data presented in the current study indicate that IEC only secrete galectin-9 upon apical exposure to CpG DNA and scGOS/lcFOS under inflammatory conditions. In endothelial cells, galectin-9 expression is regulated by the proinflammatory cytokine IFN- $\gamma$ in a PI3K/IRF3-signaling pathway, involving HDAC3 [32, 33].

Our data suggest a potential role for TLR9 signaling in the secretion of galectin- 9 . The presence of scGOS/lcFOS potentiated the effects of apical TLR9 ligation. Interestingly, scGOS/lcFOS on their own did not modulate the effector response in the IEC/PBMC coculture model. Since galectins create galectin-glycoprotein lattices on cell surfaces, the presence of scGOS/lcFOS may stabilize these lattices [26]. In addition, the extracellular domain of TLR is heavily glycosylated, which is important for proper TLR function [34]. Hence, galectin-9 may facilitate TLR9 signaling at the apical membrane of IEC and scGOS/lcFOS may contribute to effective TLR9 signaling. Further investigation on the underlying signaling pathways resulting in the release of galectin- 9 and the interactions of galectins with surface glycoproteins expressed at the membrane of IEC may provide more insight into the regulation of adaptive immune responses by IEC.

Galectin-9 is a ligand for the TIM-3 receptor expressed by $T_{h} 1, T_{h} 17$ and DC. Activation of TIM- 3 has been described to induce apoptosis of $\mathrm{T}_{h} 1$ and $\mathrm{T}_{\mathrm{h}} 17$ cells, but also 
to activate $\mathrm{DC}$ at high concentrations, which instruct naïve CD4+ T cells to produce IFN- $\gamma$, but not IL- 4 or IL-5 $[35,36]$. The present data demonstrate that galectin- 9 at $1.0 \mu \mathrm{g} / \mathrm{ml}$ induces IFN- $\gamma$ production by CD $4+\mathrm{T}$ cells only when galectin- 9 was added during moDC differentiation, whereas induction of Foxp3 expression and IL-10 production by CD4+ T cells occurred both when galectin- 9 is present during DC differentiation and during DC-Tcell interaction. Interestingly, when galectin-9 is added during moDC differentiation, these DC have the capacity to induce a $T_{h} 1$ response paralleled by a $T_{\text {reg }}$ response, which may prevent exaggerated $\mathrm{T}_{\mathrm{h}} 1$ inflammation. Galectin-9 is, in addition to the induction of T-cell apoptosis, more often associated with increased induction and expansion of $\mathrm{T}_{\text {reg }}$ cells in both allergic and inflammatory diseases [37-39]. In IEC/PBMC cocultures, IFN- $\gamma$ and IL-10 responses were abolished upon neutralization of galectin-9. Dose-response experiments using recombinant galectin-9 added to CD3/CD28-activated PBMC indeed showed that galectin- 9 can induce IFN- $\gamma$ and IL-10 production and Foxp3 expression in CD4+ T cells [29]. Furthermore, galectin- 9 secretion by IEC upon apical TLR9 ligation in the presence of scGOS/lcFOS only occurred under inflammatory conditions. This may be the consequence of low TLR9 expression by IEC in the absence of inflammatory conditions [12].

In an allergic setting, galectin-9 secretion by IEC may play an important role in suppressing $\mathrm{T}_{\mathrm{h}} 2$-associated cytokine production. It currently remains unclear whether the reduction in IL-13 secretion by CpG DNA and scGOS/lcFOS depends on galectin-9 as well. However, the current data implicate that galectin-9 is involved in regu- lating immune responses by TLR9-exposed IEC in the presence or absence of scGOS/lcFOS. Furthermore, suppression of IL-13 secretion also occurred in the absence of IEC. This may suggest that, besides IEC, PBMC may also produce galectin-9. In fact, IEC may secrete galectin-9 and thereby instruct immune cells to produce galectins as well. This phenomenon has been described for intestinal epithelium-derived thymic stromal lymphopoietin, too [40]. Future studies are warranted to investigate the involvement of galectin-9 in regulating $\mathrm{T}_{\mathrm{h}} 2$-polarized immune responses.

In summary, the present in vitro experiments show a potential role for apical TLR9 ligation of IEC in the presence scGOS/lcFOS in supporting IFN- $\gamma$ - and IL-10-mediated effector responses. Furthermore, $\mathrm{T}_{\mathrm{h}} 2$-associated cytokine production was also reduced, implicating the involvement of nondigestible oligosaccharides in suppressing allergy. Galectin- 9 secreted by IEC under inflammatory conditions supported IFN- $\gamma$ and IL-10 production acting on APC. Regulation of galectins expressed in the intestine may be a worthwhile approach to prevent or treat allergic and inflammatory diseases of the gastrointestinal tract.

\section{Acknowledgments}

We would like to thank Jan Knol for providing the genomic DNA derived from B. breve M-16V and Saskia Overbeek for her assistance in the cell culture experiments. This study was financially supported and performed within the framework of the Dutch Top Institute Pharma, project T1-214.

\section{References}

1 Cardoso CR, Teixeira G, Provinciatto PR, Godoi DF, Ferreira BR, Milanezi CM, Ferraz DB, Rossi MA, Cunha FQ, Silva JS: Modulation of mucosal immunity in a murine model of food-induced intestinal inflammation. Clin Exp Allergy 2008;38:338-349.

2 Artis D: Epithelial-cell recognition of commensal bacteria and maintenance of immune homeostasis in the gut. Nat Rev Immunol 2008;8:411-420.

3 Iliev ID, Spadoni I, Mileti E, Matteoli G, Sonzogni A, Sampietro GM, Foschi D, Caprioli F, Viale G, Rescigno M: Human intestinal epithelial cells promote the differentiation of tolerogenic dendritic cells. Gut 2009;58: 1481-1489.
4 Uehara A, Fujimoto Y, Fukase K, Takada H: Various human epithelial cells express functional Toll-like receptors, NOD1 and NOD2 to produce anti-microbial peptides, but not proinflammatory cytokines. Mol Immunol 2007;44:3100-3111.

-5 Singh JC, Cruickshank SM, Newton DJ, Wakenshaw L, Graham A, Lan J, Lodge JP, Felsburg PJ, Carding SR: Toll-like receptor-mediated responses of primary intestinal epithelial cells during the development of colitis. Am J Physiol Gastrointest Liver Physiol 2005; 288:G514-G524.

6 Abreu MT, Arnold ET, Thomas LS, Gonsky R, Zhou Y, Hu B, Arditi M: TLR4 and MD-2 expression is regulated by immune-mediated signals in human intestinal epithelial cells. J Biol Chem 2002;277:20431-20437.
7 Ewaschuk JB, Backer JL, Churchill TA, Obermeier F, Krause DO, Madsen KL: Surface expression of Toll-like receptor 9 is upregulated on intestinal epithelial cells in response to pathogenic bacterial DNA. Infect Immun 2007;75:2572-2579.

8 Abreu MT, Vora P, Faure E, Thomas LS, Arnold ET, Arditi M: Decreased expression of Toll-like receptor-4 and MD-2 correlates with intestinal epithelial cell protection against dysregulated proinflammatory gene expression in response to bacterial lipopolysaccharide. J Immunol 2001;167:16091616.
scGOS/lcFOS Supports $\mathrm{T}_{\mathrm{h}} 1 / \mathrm{T}_{\text {reg }}$

Responses via Galectin-9
J Innate Immun 2013;5:625-638 DOI: $10.1159 / 000350515$ 
9 Melmed G, Thomas LS, Lee N, Tesfay SY, Lukasek K, Michelsen KS, Zhou Y, Hu B, Arditi M, Abreu MT: Human intestinal epithelial cells are broadly unresponsive to Toll-like receptor 2-dependent bacterial ligands: implications for host-microbial interactions in the gut. J Immunol 2003;170:1406-1415.

10 Lee J, Mo JH, Katakura K, Alkalay I, Rucker AN, Liu YT, Lee HK, Shen C, Cojocaru G, Shenouda S, Kagnoff M, Eckmann L, BenNeriah Y, Raz E: Maintenance of colonic homeostasis by distinctive apical TLR9 signalling in intestinal epithelial cells. Nat Cell Biol 2006;8:1327-1336.

11 de Kivit S, van Hoffen E, Korthagen N, Garssen J, Willemsen LE: Apical TLR ligation of intestinal epithelial cells drives a Th1-polarized regulatory or inflammatory type effector response in vitro. Immunobiology 2011;216: 518-527.

-12 Zhu FG, Kandimalla ER, Yu D, Agrawal S: Oral administration of a synthetic agonist of Toll-like receptor 9 potently modulates peanut-induced allergy in mice. J Allergy Clin Immunol 2007;120:631-637.

13 Iliev ID, Mileti E, Matteoli G, Chieppa M, Rescigno M: Intestinal epithelial cells promote colitis-protective regulatory T-cell differentiation through dendritic cell conditioning. Mucosal Immunol 2009;2:340-350.

$\checkmark 14$ Hougee S, Vriesema AJ, Wijering SC, Knippels LM, Folkerts G, Nijkamp FP, Knol J, Garssen J: Oral treatment with probiotics reduces allergic symptoms in ovalbumin-sensitized mice: a bacterial strain comparative study. Int Arch Allergy Immunol 2010;151: 107-117.

15 van Hoffen E, Korthagen NM, de Kivit S, Schouten B, Bardoel B, Duivelshof A, Knol J, Garssen J, Willemsen LE: Exposure of intestinal epithelial cells to UV-killed Lactobacillus GG but not Bifidobacterium breve enhances the effector immune response in vitro. Int Arch Allergy Immunol 2010;152:159-168.

16 Bouladoux N, Hall JA, Grainger JR, Dos Santos LM, Kann MG, Nagarajan V, Verthelyi D, Belkaid Y: Regulatory role of suppressive motifs from commensal DNA. Mucosal Immunol 2012;5:623-634.

17 Campeau JL, Salim SY, Albert EJ, Hotte N, Madsen KL: Intestinal epithelial cells modulate antigen-presenting cell responses to bacterial DNA. Infect Immun 2012;80:26322644.

-18 Schouten B, van Esch BC, Hofman GA, van Doorn SA, Knol J, Nauta AJ, Garssen J, Willemsen LE, Knippels LM: Cow milk allergy symptoms are reduced in mice fed dietary synbiotics during oral sensitization with whey. J Nutr 2009;139:1398-1403.
19 van der Aa LB, Heymans HS, van Aalderen WM, Sillevis Smitt JH, Knol J, Ben Amor K, Goossens DA, Sprikkelman AB, Synbad Study Group: Effect of a new synbiotic mixture on atopic dermatitis in infants: a randomized-controlled trial. Clin Exp Allergy 2010;40:795-804.

20 Field CJ: The immunological components of human milk and their effect on immune development in infants. J Nutr 2005;135:1-4.

21 Newburg DS: Oligosaccharides in human milk and bacterial colonization. J Pediatr Gastroenterol Nutr 2000;30(suppl 2):S8-S17.

-22 Hirabayashi J, Hashidate T, Arata Y, Nishi N, Nakamura T, Hirashima M, Urashima T, Oka T, Futai M, Muller WE, Yagi F, Kasai K: Oligosaccharide specificity of galectins: a search by frontal affinity chromatography. Biochim Biophys Acta 2002;1572:232-254.

23 de Kivit S, Kraneveld AD, Garssen J, Willemsen LE: Glycan recognition at the interface of the intestinal immune system: target for immune modulation via dietary components. Eur J Pharmacol 2011;668(suppl 1):S124S132.

24 Nio-Kobayashi J, Takahashi-Iwanaga H, Iwanaga T: Immunohistochemical localization of six galectin subtypes in the mouse digestive tract. J Histochem Cytochem 2009;57:41-50.

25 Wada J, Kanwar YS: Identification and characterization of galectin-9, a novel beta-galactoside-binding mammalian lectin. J Biol Chem 1997;272:6078-6086.

26 van Kooyk Y, Rabinovich GA: Protein-glycan interactions in the control of innate and adaptive immune responses. Nat Immunol 2008;9: 593-601.

27 Rabinovich GA, Toscano MA: Turning 'sweet' on immunity: galectin-glycan interactions in immune tolerance and inflammation. Nat Rev Immunol 2009;9:338-352.

28 Wilson K: Preparation of genomic DNA from bacteria. Curr Protoc Mol Biol 2001;chapt 2:unit 2.4.

29 de Kivit S, Saeland E, Kraneveld AD, van de Kant HJ, Schouten B, van Esch BC, Knol J, Sprikkelman AB, van der Aa LB, Knippels LM, Garssen J, van Kooyk Y, Willemsen LE: Galectin- 9 induced by dietary synbiotics is involved in suppression of allergic symptoms in mice and humans. Allergy 2012;67:343-352.

30 Garcia-Vallejo JJ, Van Het Hof B, Robben J, Van Wijk JA, Van Die I, Joziasse DH, Van Dijk W: Approach for defining endogenous reference genes in gene expression experiments. Anal Biochem 2004;329:293-299.
31 Bogunovic M, Ginhoux F, Helft J, Shang L, Hashimoto D, Greter M, Liu K, Jakubzick C, Ingersoll MA, Leboeuf M, Stanley ER, Nussenzweig M, Lira SA, Randolph GJ, Merad M: Origin of the lamina propria dendritic cell network. Immunity 2009;31:513-525.

32 Imaizumi T, Yoshida H, Nishi N, Sashinami H, Nakamura T, Hirashima M, Ohyama C, Itoh K, Satoh K: Double-stranded RNA induces galectin-9 in vascular endothelial cells: involvement of TLR3, PI3K, and IRF3 pathway. Glycobiology 2007;17:12C-15C.

33 Alam S, Li H, Margariti A, Martin D, Zampetaki A, Habi O, Cockerill G, Hu Y, Xu Q, Zeng L: Galectin-9 protein expression in endothelial cells is positively regulated by histone deacetylase 3. J Biol Chem 2011;286: 44211-44217.

- 34 Amith SR, Jayanth P, Franchuk S, Finlay T, Seyrantepe V, Beyaert R, Pshezhetsky AV, Szewczuk MR: Neu1 desialylation of sialyl alpha-2,3-linked beta-galactosyl residues of Toll-like receptor 4 is essential for receptor activation and cellular signaling. Cell Signal 2010;22:314-324.

35 Zhu C, Anderson AC, Schubart A, Xiong H, Imitola J, Khoury SJ, Zheng XX, Strom TB, Kuchroo VK: The Tim-3 ligand galectin-9 negatively regulates $\mathrm{T}$ helper type 1 immunity. Nat Immunol 2005;6:1245-1252.

- 36 Dai SY, Nakagawa R, Itoh A, Murakami H, Kashio Y, Abe H, Katoh S, Kontani K, Kihara M, Zhang SL, Hata T, Nakamura T, Yamauchi A, Hirashima M: Galectin-9 induces maturation of human monocyte-derived dendritic cells. J Immunol 2005;175:2974-2981.

- 37 Katoh S, Oomizu S, Niki T, Shimizu H, Obase Y, Korenaga M, Oka M, Hirashima M: Possible regulatory role of galectin-9 on Ascaris suum-induced eosinophilic lung inflammation in mice. Int Arch Allergy Immunol 2012; 158(suppl 1):58-65.

- 38 Lv K, Zhang Y, Zhang M, Zhong M, Suo Q: Galectin-9 ameliorates Con A-induced hepatitis by inducing $\mathrm{CD} 4(+) \mathrm{CD} 25$ (low/int) effector T-cell apoptosis and increasing regulatory T cell number. PLoS One 2012; 7:e48379.

-39 Seki M, Oomizu S, Sakata KM, Sakata A, Arikawa T, Watanabe K, Ito K, Takeshita K, Niki T, Saita N, Nishi N, Yamauchi A, Katoh S, Matsukawa A, Kuchroo V, Hirashima M: Galectin-9 suppresses the generation of Th17, promotes the induction of regulatory $\mathrm{T}$ cells, and regulates experimental autoimmune arthritis. Clin Immunol 2008;127:78-88.

40 Spadoni I, Iliev ID, Rossi G, Rescigno M: Dendritic cells produce TSLP that limits the differentiation of Th17 cells, fosters Treg development, and protects against colitis. Mucosal Immunol 2012;5:184-193. 